\title{
Histone Deacetylase Inhibition has Cardiac and Vascular Protective Effects in Rats With Pressure Overload Cardiac Hypertrophy
}

\author{
H. JUNG ${ }^{1^{*}}$, E. LEE $\mathrm{LE}^{2,3,4,5^{*}}$, I. KIM $\mathrm{KM}^{2,3,4,5}$, J. H. SONG ${ }^{6}$, G. J. KIM ${ }^{1}$ \\ * These authors contributed equally to this work.
}

${ }^{1}$ Department of Thoracic and Cardiovascular Surgery, School of Medicine, Kyungpook National University, Kyungpook National University Hospital, Daegu, Republic of Korea, ${ }^{2}$ Department of Pharmacology, School of Medicine, Kyungpook National University, Daegu, Republic of Korea, ${ }^{3}$ BK21 PLUS KNU Biomedical Convergence Program, School of Medicine, Kyungpook National University, Daegu, Republic of Korea, ${ }^{4}$ Cardiovascular Research Institute, Kyungpook National University, Daegu, Republic of Korea, ${ }^{5}$ Cell and Matrix Research Institute, Kyungpook National University, Daegu, Republic of Korea, ${ }^{6}$ Division of Public Health Medical Service, Kyungpook National University Hospital, Daegu, Republic of Korea

Received December 26, 2018

Accepted June 3, 2019

Epub Ahead of Print August 19, 2019

\section{Summary}

Histone deacetylase (HDAC) inhibitors have shown beneficial effects in animal models of cardiovascular diseases. We hypothesized that HDAC inhibitor, sodium valproate (VPA), has cardiac and vascular protective effects in rats with pressure overload cardiac hypertrophy induced by transverse aortic constriction (TAC). Sections of the heart were visualized after hematoxylin and eosin staining, picrosirius red staining and immunohistochemistry. The expression of genes related to cardiac hypertrophy, fibrosis, and oxidative stress was determined by quantitative real-time polymerase chain reaction. The aortic ring tension analysis was conducted using both the ascending aorta and descending thoracic aorta. TAC increased the expression of hypertrophic, fibrotic, and oxidative stress genes, which was attenuated by VPA. In the ascending aorta with intact endothelium, there was a significant decrease in the relaxation response, which was recovered by VPA treatment. These results indicate that VPA has cardiac and vascular protective effects in rats with pressure overload cardiac hypertrophy.

\section{Key words}

Cardiac hypertrophy • Fibrosis • Histone deacetylase inhibitors • Oxidative stress • Vascular endothelium • Ventricular remodeling

\section{Corresponding author}

G. J. Kim, Department of Thoracic and Cardiovascular Surgery, Kyungpook National University Hospital, Kyungpook National University, 130 Dongdeok-ro, Jung-gu, Daegu 41944, Republic of Korea. E-mail: straightroot@knu.ac.kr

\section{Introduction}

Heart failure is a serious consequence of cardiac hypertrophy, and it has no cure with current therapies, which only aim at delaying the disease progression (Ooi et al. 2015). Cardiac remodeling initially occurs by compensatory response to increased hemodynamic pressure overload, which initially alters the heart performance. This gradually decreases ventricular compliance together with cardiac hypertrophy in the left ventricle (LV) and endothelial dysfunction, ultimately exceeds the limit of compensation, and eventually leads to heart failure (Iyer et al. 2010). Thus, there is a continuous effort to identify substances that can prevent or reverse cardiac remodeling in heart disease (Simko et al. 2017).

Several studies have proved that angiotensinconverting enzyme inhibitors and angiotensin II type 1 receptor blockers improve hemodynamics under heart disease conditions by the dilatation of peripheral blood 
vessels, eventually reducing afterload. However, these effects might be caused by the indirect effects on blood vessels, instead of direct regulation of cardiac remodeling (Chen et al. 1998). Histone deacetylase (HDAC) inhibitors, currently used as anti-tumor agents, act by the direct regulation of cardiac protective effect, providing a novel therapeutic strategy for heart diseases. Studies have shown the ability of various HDAC inhibitors in different cardiac hypertrophic animal models. Trichostatin A, sodium valproate (VPA), and SK-7041 block cardiac hypertrophy in angiotensin II infusioninduced cardiac hypertrophic mice or rats (Kee et al. 2006). VPA attenuates cardiac remodeling in spontaneously hypertensive rats (Cardinale et al. 2010, Kang et al. 2015). VPA (Iyer et al. 2010, Kee et al. 2013) and CG200475 (Lee et al. 2016) suppress cardiac hypertrophy and fibrosis in DOCA-salt hypertensive rats. Palmatine (Yuan et al. 2017) and tubacin (Tao et al. 2016) attenuate isoprenaline-induced cardiac hypertrophic rats.

Both classes of HDACs, class I (1, 2, 3, and 8) and class IIa (4, 5, 7, and 9), are associated with cardiac hypertrophy; however, these HDACs perform completely opposite roles (Yoon and Eom 2016). The roles of the HDAC inhibitors in cardiac hypertrophy are being widely studied. For example, the expression of HDAC2 is increased by palmatine in isoprenaline-induced hypertrophic rat model (Yuan et al. 2017). In DOCA-salt induced hypertrophic heart rat model, the enzyme activity of HDAC6 and HDAC8 was up-regulated, and their activities were inhibited by VPA (Kee et al. 2013). Moreover, in hypertrophied heart of spontaneously hypertensive rats, VPA administration lowered global HDAC activity level compared with that in the respective controls (Cardinale et al. 2010). HDAC inhibitors also effectively block cardiac fibrosis. Mocetinostat (MGCD0103), class I HDAC and HDAC11 inhibitor, reverse cardiac fibrosis in congestive heart failure model (Nural-Guvener et al. 2014). When treated with tubacin, a class IIb HDAC (6 and 10) inhibitor, the expression of cardiac fibrosis marker genes was attenuated in isoprenaline-induced heart dysfunction rats (Tao et al. 2016). HDAC inhibitors show many beneficial effects that control heart failures. However, the definite mechanisms of these effects remain elusive (McKinsey 2011). Taken together, pan-HDAC inhibitors are helpful for improving cardiac hypertrophy and fibrosis.

VPA, a short-chain branched fatty acid HDAC inhibitor, is an anticonvulsant that is clinically widely used in the treatment of seizure, migraine, and bipolar disorder (Gurvich et al. 2004). Therefore, it will not be difficult to extend the therapeutic application of VPA to cardiovascular diseases (Cho et al. 2010). Spontaneously hypertensive rats, Dahl salt sensitive rats, and pharmacologically induced (such as DOCA-salt hypertensive rats, angiotensin II infusion rats and isoprenaline-induced cardiac hypertrophic rats) cardiac hypertrophic animal models take long period of time to progress cardiac hypertrophy (Gomes et al. 2013). Meanwhile, transverse aortic constriction (TAC) with well-established surgical technique induces pressure overload in the LV, and it was first described by Rockman et al. (1991). The sudden onset of systemic hypertension achieved with TAC results in the rapid development of LV hypertrophy, allowing the examination of influence of pharmacological or molecular interventions (Tavakoli et al. 2017). HDAC inhibitor Trichostatin A (Ooi et al. 2015) and sodium phenylbutyrate (Ma et al. 2016) have been examined in TAC-induced pressure overload cardiac hypertrophic rats.

In the present study, we hypothesized that HDAC inhibitor, VPA, has cardiac and vascular protective effects in the rat with pressure overload cardiac hypertrophy induced by TAC.

\section{Methods}

Animals

The investigation was conducted in accordance with the National Institutes of Health Guide for the Care and Use of Laboratory Animals and was approved by the Institutional Review Board of Kyungpook National University. Male Sprague-Dawley rats were housed in a cage at $20-23^{\circ} \mathrm{C}$ with a 12 -h light/dark cycle. Minimally invasive TAC was performed by the following protocol (Tavakoli et al. 2017). Ten-week-old rats, weighing $350-400 \mathrm{~g}$, were placed in the supine position under the effect of anesthesia containing a mixture of ketamine $(150 \mathrm{mg} / \mathrm{kg}$, intraperitoneally; Yuhan, Seoul, Republic of Korea and xylazine $(18 \mathrm{mg} / \mathrm{kg}$, intraperitoneally; Bayer, Seoul, Republic of Korea). Under sterile conditions, partial sternotomy was carefully performed not to rip the mediastinum pleura. The transverse aorta was tied with 5-0 silk suture using a 22-gauge needle between the innominate artery and left common carotid artery. The needle was then immediately removed, forming a constricted transverse aorta (Luo et al. 2015). Sham-operated (sham) rats were subjected to the same procedure without tying the transverse aorta. 
After TAC and sham operation, the sternum was closed, and the rats were allowed to recover overnight before being randomly assigned to one of the following four groups: sham $(n=6)$, sham plus VPA $(n=6)$, TAC $(n=6)$, and TAC plus VPA $(n=6)$. The rats had free access to drinking water with or without $0.71 \%$ VPA. The rats were euthanized using pentobarbital sodium (ENTOBAR $^{\circledR}, \quad 0.5 \mathrm{ml} / \mathrm{kg}, \quad$ intraperitoneally; Hanlim Pharm Co., Seoul, Republic of Korea). The weight of wet heart was measured and normalized against the length of tibia. The aorta was immediately excised for the organ bath experiment. The tissues were frozen in liquid nitrogen and stored at $-80{ }^{\circ} \mathrm{C}$ until further analysis.

Drug

VPA was purchased from Sigma-Aldrich (St. Louis, MO, USA).

\section{Blood pressure measurement}

The blood pressure of the rats was measured by the tail-cuff method. The rats were preheated on a hotplate at $35{ }^{\circ} \mathrm{C}$ for $10 \mathrm{~min}$ and then placed in plastic restrainers. A cuff with a pneumatic pulse sensor was attached to the tail. The blood pressure of the rats was recorded using a non-invasive blood pressure controller system $\left(\mathrm{CODA}^{\circledR}\right.$ High Throughput Monitor, Kent Scientific Co., Torrington, CT, USA). The values are presented as the average of at least five consecutive readings obtained from each rat.

\section{Aorta preparation and tension recording}

The excised thoracic aorta was placed in modified Krebs solution, containing (in mmol/l) $\mathrm{NaCl}$, 115.0; KCl, 4.7; $\mathrm{CaCl}_{2}, 2.5 ; \mathrm{MgCl}_{2}, 1.2 ; \mathrm{NaHCO}_{3}, 25.0$; $\mathrm{KH}_{2} \mathrm{PO}_{4}, 1.2$; and glucose, 10.0. The connective tissue adhering to the aorta was cleaned on wet filter paper, soaked in Krebs solution, and cut into four ring segments (4.0 $\mathrm{mm}$ long). Some rings were denuded of endothelium by gently rubbing the internal surface with the edge of a forcep. Two stainless steel triangles were inserted through each vessel ring. Each aortic ring was suspended in a water-jacketed organ bath $(20 \mathrm{ml})$ maintained at $37{ }^{\circ} \mathrm{C}$ and aerated with a mixture of $95 \% \mathrm{O}_{2}$ and $5 \%$ $\mathrm{CO}_{2}$. One triangle was anchored to a stationary support and the other was connected to an isometric force transducer (Grass FT03C, Quincy, MA, USA). The rings were stretched to an optimal resting tension of $2.0 \mathrm{~g}$, which was maintained throughout the experiment. Each ring was equilibrated in the organ bath solution for $90 \mathrm{~min}$ before the evaluation of contractile response after the addition of $50 \mathrm{mmol} / \mathrm{K} \mathrm{KCl}$. Isometric responses were recorded using a computerized data acquisition system (PowerLab/8SP; AD Instruments, Castle Hill, NSW, Australia). Cumulative contractile responses were determined after the serial addition of phenylephrine (PE). Cumulative vasorelaxant responses were determined in the aortic rings with or without endothelium by the serial addition of acetylcholine (ACh) or sodium nitroprusside (SNP), respectively.

\section{Histology}

The heart tissues were fixed overnight in $4 \%$ formalin, dehydrated, and embedded in paraffin following the conventional method. The paraffinembedded samples were sectioned to $3 \mu \mathrm{m}$ thickness. The sections were stained with hematoxylin and eosin, and picrosirius red. For immunohistochemistry, the slides were incubated with anti-atrial natriuretic peptide (ANP, Abcam, Cambridge, UK) and anti-brain natriuretic peptide (BNP, Abcam, Cambridge, UK).

The whole slides were examined using digital slide scanner (Pannoramic SCAN II, 3DHISTECH Ltd., Budapest, Hungary) and digital slide images were viewed and analyzed by CaseViewer (3DHISTECH Ltd., Budapest, Hungary). The slides were also examined using a light microscope (Axioplan 2 imaging, ZEISS, Jena, Thuringia, Germany) and the slide images were then digitalized and transformed into computerized images. The cross-sectional areas were calculated by an automatic area quantification program in ImageJ software (http://rsbweb.nih.gov).

\section{Quantitative real-time polymerase chain reaction}

Quantitative real-time polymerase chain reaction (qRT-PCR) was performed to detect the expression of ANP (Nppa) and BNP (Nppb) as markers for cardiac hypertrophy; Collagen-1, -3, and Fibronectin as markers for cardiac fibrosis; and NADPH oxidase subunits-1, -2, and -4 (Nox-1, -2 , and -4 ) as markers for oxidative stress. The tissues (about $100 \mathrm{mg}$ ) were homogenized in liquid nitrogen with a glass homogenizer. The RNA was extracted using QIAzol ${ }^{\circledR}$ Lysis Reagent (QIAGEN science, Germantown, MD, USA) according to manufacturer's instructions. The total RNA $(2 \mu \mathrm{g})$ was reverse-transcribed into cDNA using the Thermo Scientific $^{\mathrm{TM}}$ RevertAid $^{\mathrm{TM}}$ first strand cDNA kit (Fermentas, Vilnius, Lithuania, EU) in a reaction mixture of volume $20 \mu \mathrm{l}$, according to the manufacturer's instructions. qRT-PCR was performed using the ABI Prism 7000 sequence detection system (Applied 
Biosystems, Foster City, CA, USA). The reaction mixture contained $10 \mu 1$ of SYBR Green PCR $2 \times$ master mix (CellSafe, Yongin-si, Kyounggi-do, Republic of Korea), $4 \mu \mathrm{l}$ of cDNA, and $200 \mu \mathrm{l}$ of primer set (Table S1). All samples were amplified in triplicates in a 96-well plate and the cycling conditions were as follows: $2 \mathrm{~min}$ at $50{ }^{\circ} \mathrm{C}, 10 \mathrm{~min}$ at $95^{\circ} \mathrm{C}$, and 40 cycles at $95^{\circ} \mathrm{C}$ for $15 \mathrm{~s}$ followed by $1 \mathrm{~min}$ at $60^{\circ} \mathrm{C}$. The relative mRNA expression level was determined by calculating the values of $\Delta$ cycle threshold $(\Delta \mathrm{Ct})$ by normalizing the average $\mathrm{Ct}$ value compared with its endogenous control (Gapdh), and then calculating the $2^{-\Delta \Delta \mathrm{Ct}}$ value.

\section{Echocardiography}

Cardiac remodeling was evaluated by echocardiography using a $15 \mathrm{MHz}$ linear array transducer (S-sharp, New Taipei City, Taiwan). The rats were anesthetized with gas inhalation of isoflurane (1-3\%, Hana Pharm. Co. Ltd., Hwaseong-si, Kyounggi-do, Republic of Korea) during echocardiographic examinations. The $\mathrm{M}$-mode images from the parasternal long axis view were used to measure the conventional echocardiographic parameters. The internal dimension of left ventricle (LVID), thickness of left ventricular posterior wall (LVPW), and thickness of interventricular septum (IVS) were measured at the end-systole as structural parameters.

For functional parameters, fractional shortening (FS) was calculated as percent change in LV dimension, $\mathrm{FS}=[($ LVIDd - LVIDs $) /$ LVIDd $] \times 100 ;$ where, LVIDd is the internal dimension of LV at end-diastole. The ejection fraction $(\mathrm{EF})$ was calculated as percent change in $\mathrm{LV}$ volume, $\mathrm{EF}=\left[\left(\mathrm{LVIDd}^{2}-\mathrm{LVIDs}^{2}\right) / \mathrm{LVIDd}^{2}\right] \times 100$.

\section{Statistics}

The results are expressed as mean \pm standard error. Kruskal-Wallis test and the one-way analysis of variance (ANOVA), followed by post hoc Tukey's comparison test were performed to analyze the data. Differences were considered significant at $P<0.05$. Student's $t$-test was applied for the analysis of significant differences between two groups. The procedures were performed using SPSS software (23.0, SPSS Inc., Chicago, IL, USA).

\section{Results}

\section{VPA attenuates hypertension}

Using the tail-cuff method, systolic blood pressure was measured and recorded for four weeks. TAC resulted in significantly increased systolic blood pressure about $40 \mathrm{~mm} \mathrm{Hg}$, which was attenuated about $30 \mathrm{~mm} \mathrm{Hg}$ by the administration of VPA (Fig. 1a). Neither TAC nor VPA administration affected body weight gain (Fig. 1b).

\section{VPA attenuates cardiac remodeling and improves heart} function

Echocardiogram demonstrated that whether VPA attenuates cardiac remodeling in LV. The thickness of both LVPW and IVS was significantly increased in TAC rats compared with those in the sham rats, and VPA treatment considerably attenuated the thickness of both LVPW and IVS in TAC rats (Fig. 1c, d and f). Consequently, LVID significantly decreased in TAC rats compared with that in the sham rats, and VPA administration significantly increased LVID in TAC rats (Fig. 1e).

Heart functions were also evaluated by FS (Fig. 1g) and EF (Fig. 1h). Both were significantly increased in TAC rats compared with those in the sham rats, and VPA treatment considerably decreased the FS and EF in TAC rats.

\section{VPA ameliorates cardiac hypertrophy}

The heart weight/tibia length ratio was used to show phenotypic changes related to cardiac hypertrophyinduced heart mass increase. Heart weight was significantly increased in TAC rats compared with that in the sham rats, and VPA treatment substantially attenuated the heart weight gain in TAC rats (Fig. 1i). Furthermore, the left heart weight/tibia length ratio was considerably increased in TAC rats compared with that in the sham rats, and VPA administration significantly suppressed the increase in left heart weight in TAC rats (Fig. 1j). Additionally, the right heart weight/tibia length ratio was not considerably different in TAC rats and TAC plus VPA rats (Fig. 1k).

To confirm LV hypertrophy histologically in TAC rats, we performed hematoxylin and eosin staining. The thickness of $\mathrm{LV}$ wall increased in TAC rats compared with that in the sham rats, and VPA treatment attenuated the thickness of LV wall in TAC rats (Fig. 2a). Furthermore, hypertrophy of cardiomyocyte (Fig. 2b) increased in TAC rats compared with those in the sham rats, and these effects were attenuated by VPA administration.

The expression of ANP and BNP, markers of cardiac hypertrophy, was detected by immunohistochemistry. ANP (Fig. 2c) and BNP (Fig. 2d) expression was significantly increased in TAC rats compared with those in the sham rats, which was restored by VPA administration. We also investigated the expression level 
of ANP (Nppa) and BNP (Nppb) mRNA by qRT-PCR. mRNA expression of $N p p a$ (Fig. 2e) and $N p p b$ (Fig. 2f) was increased in TAC rats, consistent with hypertrophy observed in histological evaluation. mRNA expression of $N p p a$ and Nppb was decreased with VPA treatment in TAC plus VPA rats.

\section{VPA ameliorates cardiac fibrosis}

Picrosirius red staining showed cardiac fibrosis with collagen deposition in TAC rats. Collagen deposition was increased in TAC rats compared with that in the sham rats, and VPA treatment attenuated collagen deposition in TAC plus VPA rats (Fig. 3a and b).

$$
\text { mRNA expression of Collagen-1, -3, and }
$$

Fibronectin, markers for cardiac fibrosis, was significantly increased in TAC rats compared with that in the sham rats, and VPA treatment considerably attenuated the transcription levels in TAC rats (Fig. 3c, d and e). These results were consistent with the results of histological observations.

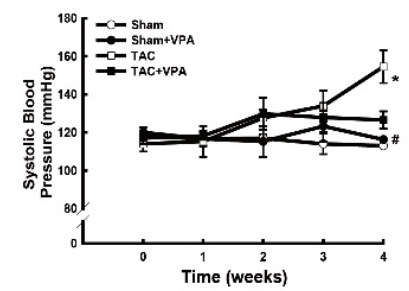

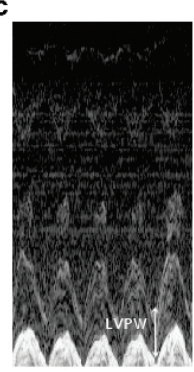

Sham

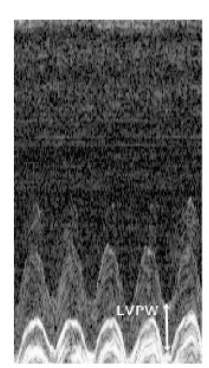

Sham + VPA

e

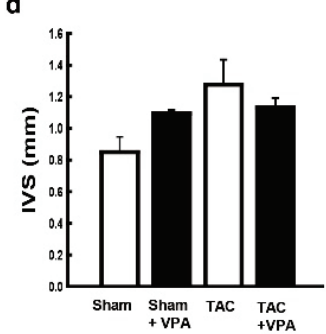

g

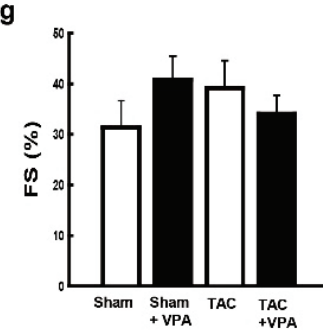

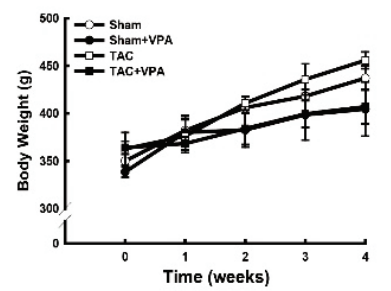

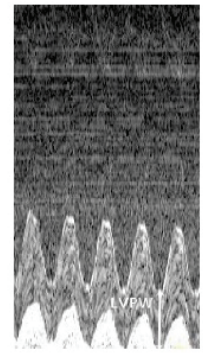

TAC

f
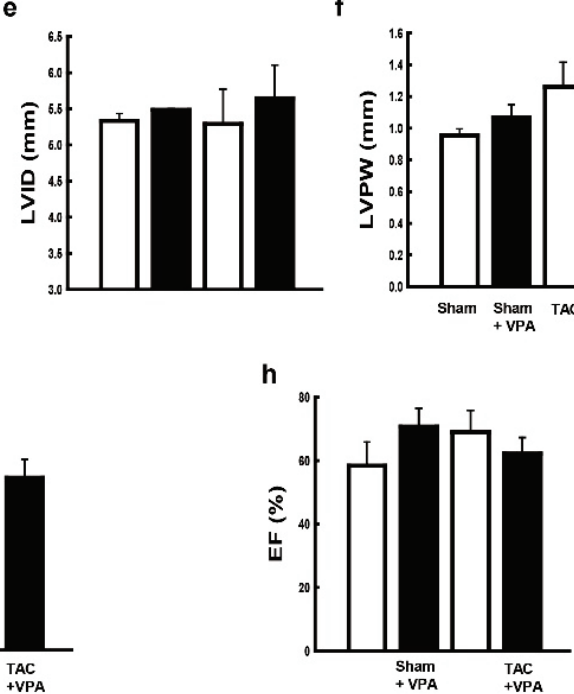
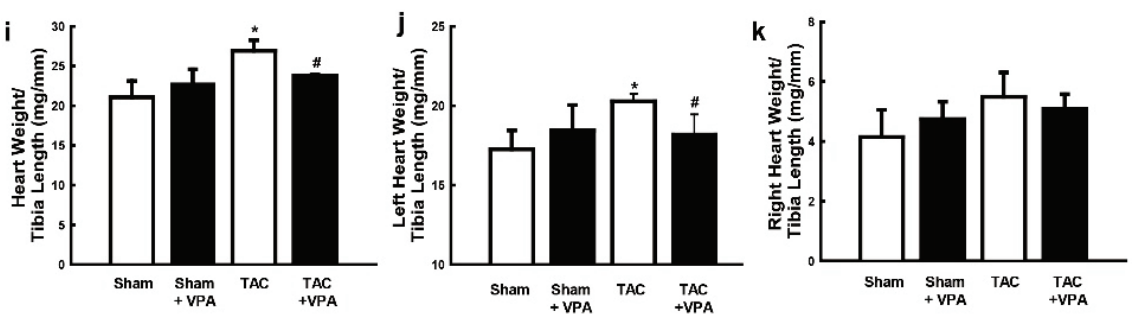

Fig. 1. Effects of VPA on systolic blood pressure, body weight, cardiac remodeling and heart function. (a) Systolic blood pressure was measured by the tail-cuff method in the sham $(n=6)$, sham plus VPA $(n=6)$, TAC $(n=6)$, and TAC plus VPA groups $(n=6)$ for four weeks. Administration of VPA attenuated TACinduced hypertension. (b) The body weight of rats was monitored for four weeks. The body weight of TAC rats treated with or without VPA were not affected. (c) Representative echocardiographic images are shown after four weeks of TAC operation with or without VPA administration. The white arrow indicates the thickness of the left ventricular posterior wall (LVPW). (d, e and f) Parameters of left ventricular remodeling, such as interventricular septal thickness (IVS, d), left ventricular internal dimension (LVID, e), and LVPW (f), were evaluated by echocardiography in the sham $(n=6)$, sham plus VPA $(n=6)$, TAC $(n=6)$, and TAC plus VPA $(n=6)$ after four weeks of TAC operation with or without VPA treatment. Parameters of the heart function, such as fractional shortening (FS, g) and ejection fraction $(E F, \mathbf{h})$, were also evaluated by echocardiography. Administration of VPA suppressed TAC-induced cardiac remodeling in left ventricle (LV) and heart function. Cardiac hypertrophy was analyzed based on heart weight/tibia length ratio. Heart weight/tibia length (i) and left heart weight/tibia length $(j)$ ratios were increased in TAC rats compared with those in the sham rats. The treatment with VPA restored heart weight/tibia length (i) and left heart weight/tibia length (j) ratios. Right heart weight/tibia length ratio (k) was similar among all the groups. VPA administration did not affect right heart weight/tibia length ratio. Data are presented as mean \pm standard error (SE) of six independent experiments $(* p<0.05$ vs. sham, ${ }^{\#} \mathrm{p}<0.05$ vs. TAC). 
a
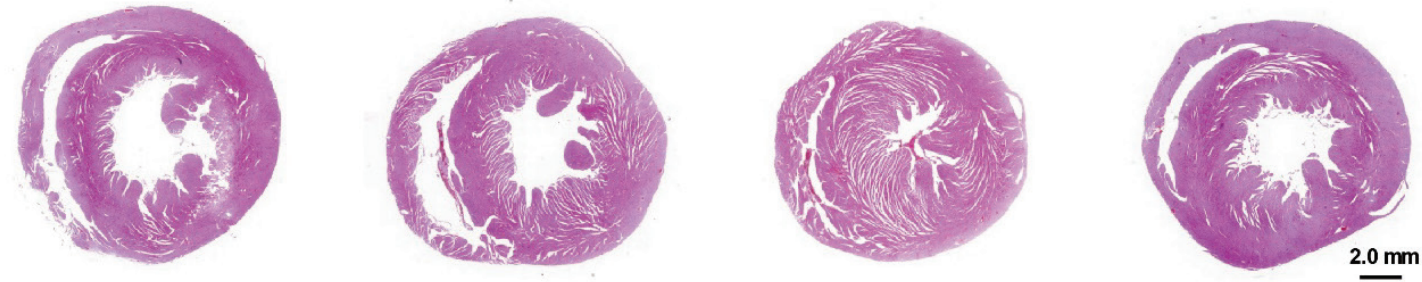

b
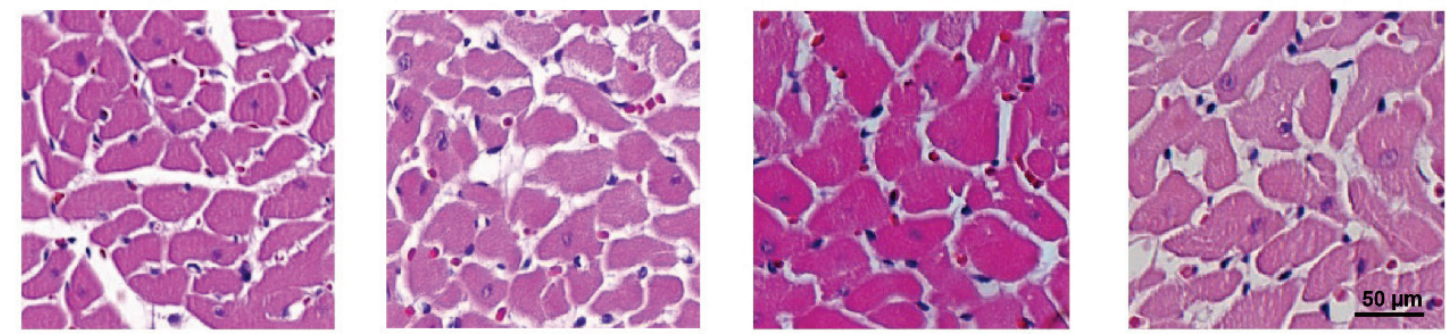

C
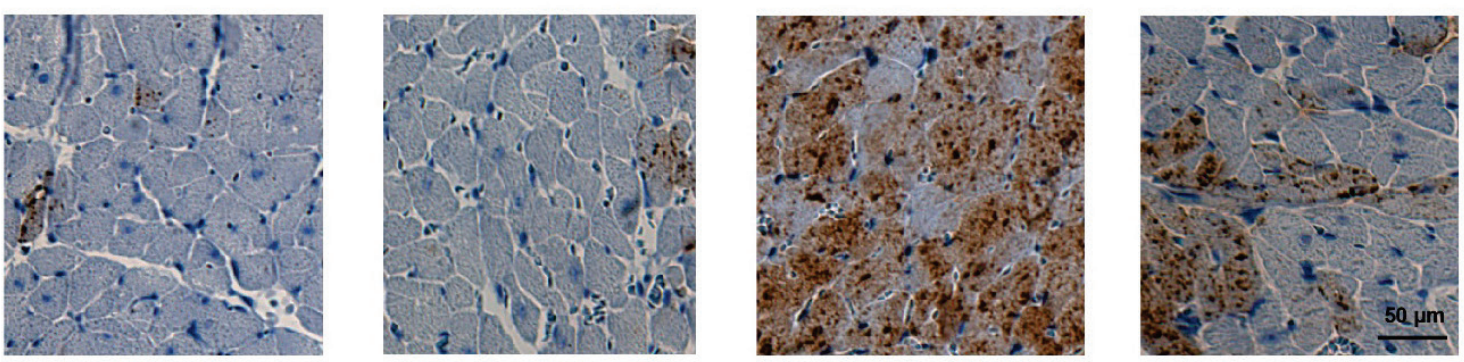

d

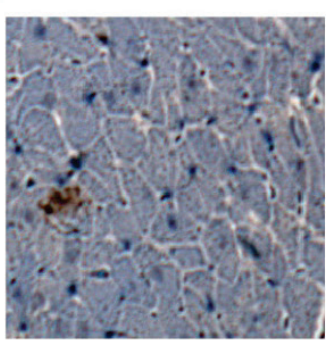

Sham

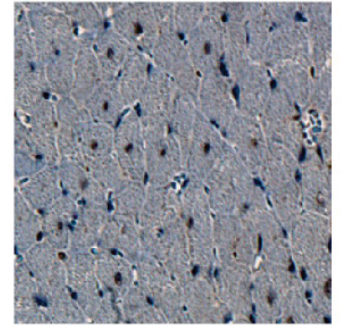

Sham + VPA

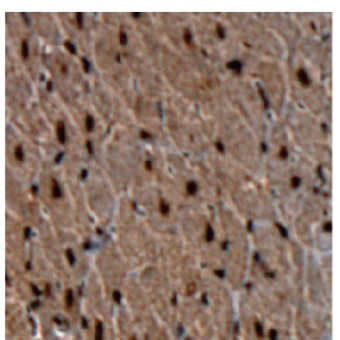

TAC

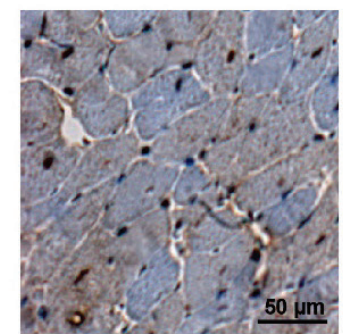

TAC + VPA
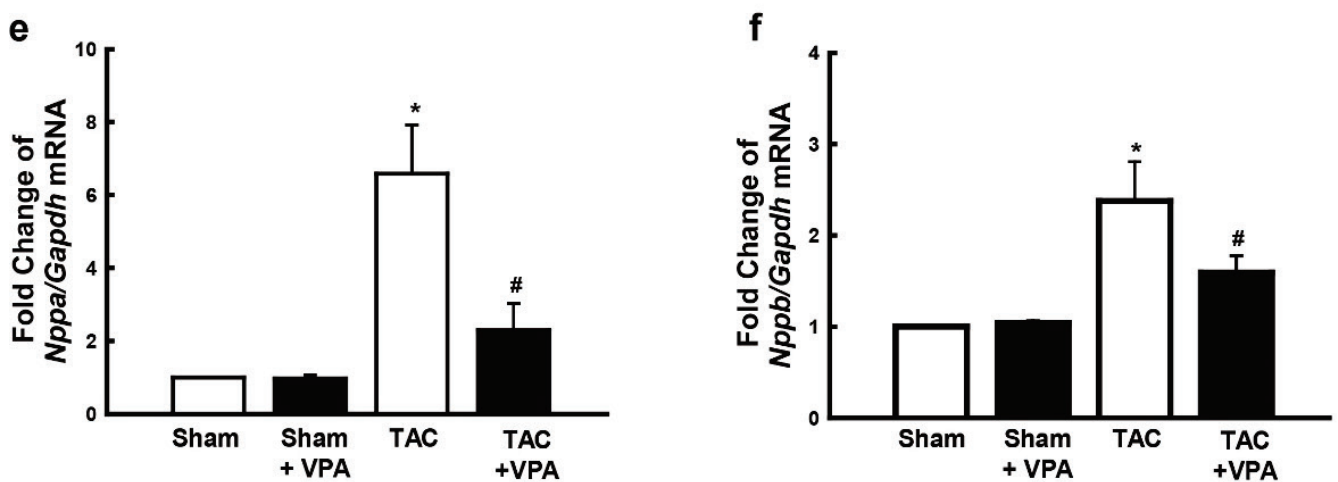

Fig. 2. Effect of VPA on cardiac hypertrophy. Histological analysis of the hearts in the sham $(n=6)$, sham plus VPA $(n=6)$, TAC ( $n=6)$, and TAC plus VPA groups $(n=6)$ was performed by hematoxylin and eosin staining. LV wall thickness (a) and hypertrophy of cardiomyocytes (b) were increased in the TAC rats compared with those in the sham rats, which were attenuated by VPA treatment. Scale bars are $2.0 \mathrm{~mm}$ (a) and $50 \mu \mathrm{m}$ (b). Immunohistochemistry of the hearts was performed using anti-ANP antibody (c) and antiBNP antibody (d). The TAC rats presented increased ANP and BNP expression (brown stain) compared with those in the sham rats, whereas, VPA treatment decreased their expression in TAC rats. Scale bar is $50 \mu \mathrm{m}$ (c and d). mRNA expression of $N p p a(\mathbf{e})$ and $N p p b(\mathbf{f})$, cardiac hypertrophy markers, was determined by quantitative real-time polymerase chain reaction (qRT-PCR). The TAC rats exhibited increased expression of $N p p a$ and $N p p b$ mRNA, which was decreased by VPA administration in TAC rats. Data are shown as mean \pm SE of six independent experiments ( ${ }^{*} p<0.05$ vs. sham, ${ }^{*} p<0.05$ vs. TAC). 
a
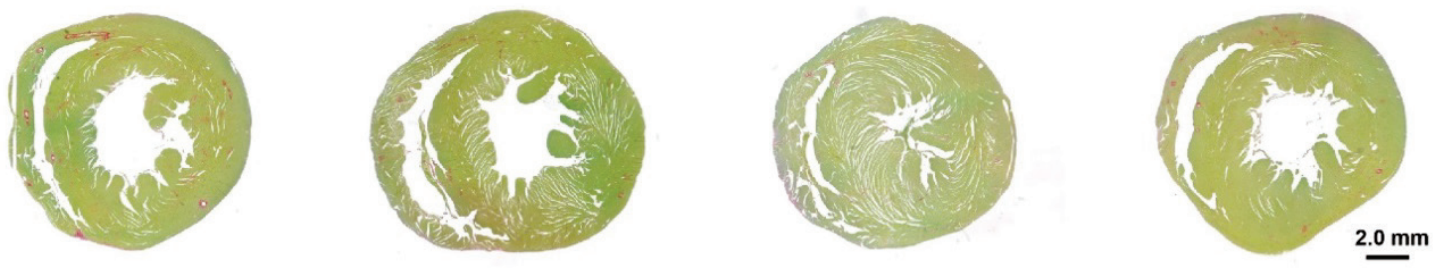

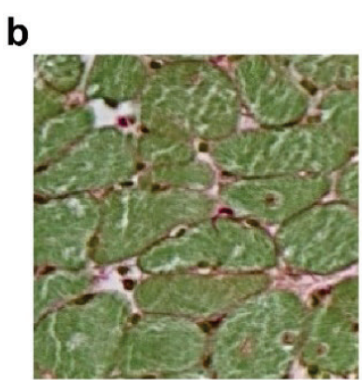

Sham

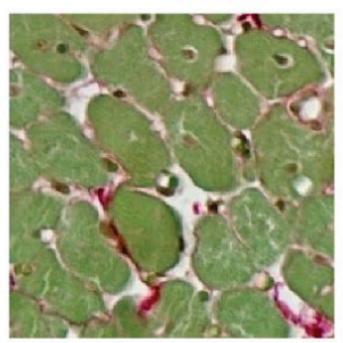

Sham + VPA

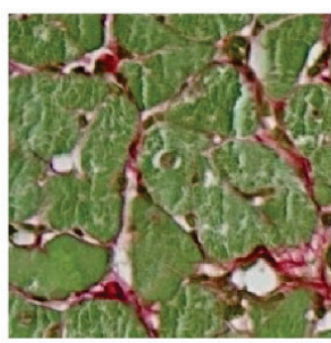

TAC

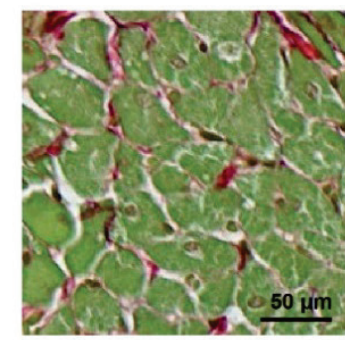

TAC + VPA
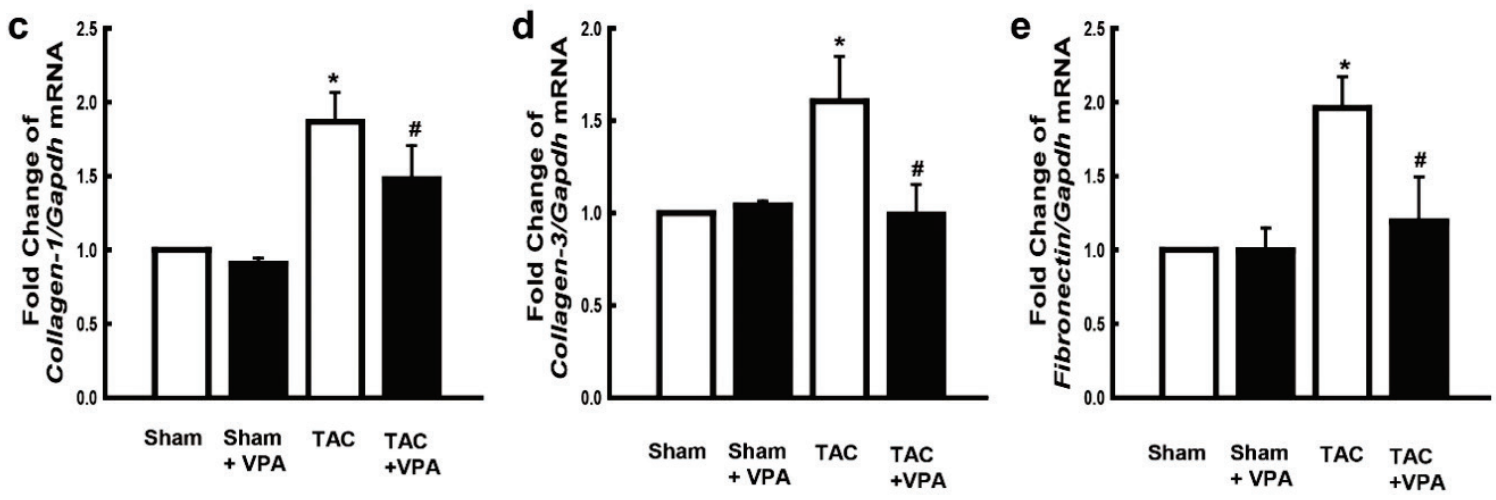

Fig. 3. Effect of VPA on cardiac fibrosis. Histological analysis of the hearts from the sham $(n=6)$, sham plus VPA ( $n=6)$, TAC ( $n=6)$, and TAC plus VPA groups $(n=6)$ was performed by picrosirius red staining. The TAC rats presented increased collagen deposition (a) and fibrosis of myocardium (b) (red stain) when compared those with the sham rats. VPA treatment attenuated cardiac fibrosis in TAC plus VPA rats. Scale bars are $2.0 \mathrm{~mm}$ (a) and $50 \mu \mathrm{m}$ (b). Gene expression of Collagen-1 (c), Collagen-3 (d), and Fibronectin (e), cardiac fibrosis markers, was determined by qRT-PCR. The TAC rats exhibited increased expression of Collagen-1, -3 , and Fibronectin mRNA, which was attenuated by VPA administration. Data are shown as mean \pm SE of six independent experiments ( $\mathrm{p}<0.05 \mathrm{vs}$. sham, ${ }^{*} \mathrm{p}<0.05 \mathrm{vs}$. TAC).

\section{VPA attenuates oxidative stress}

To determine whether oxidative stress of heart is regulated by VPA, we performed qRT-PCR. The mRNA expression of $N A D P H$ oxidase subunits-1, -2 , and -4 (Nox-1, -2, and -4), markers for oxidative stress, was significantly increased in TAC rats compared with that in the sham rats, and VPA treatment considerably attenuated the transcription level in TAC rats (Fig. 4a, b and c).

VPA improves vascular relaxation response in the ascending aorta

To evaluate whether VPA affects vascular contraction and relaxation, we conducted organ bath experiment. We investigated the contraction of aorta by treating it with PE cumulatively and relaxation of aorta by treating it with ACh cumulatively. The ascending aorta was separately evaluated by the organ bath experiment. In the ascending aorta with intact endothelium, there was a significant decrease in the relaxation response in TAC rats, and VPA treatment considerably increased the rela-xation response in TAC rats (Fig. 5b) but did not affect vascular contraction (Fig. 5a). In the descending thoracic aorta, which is usually used in conventional experiments, TAC and VPA administration did not affect vascular contraction and relaxation, irrespective of whether endo-thelium was intact (Fig. 5c and d) or denuded (Fig. 5e and f). 

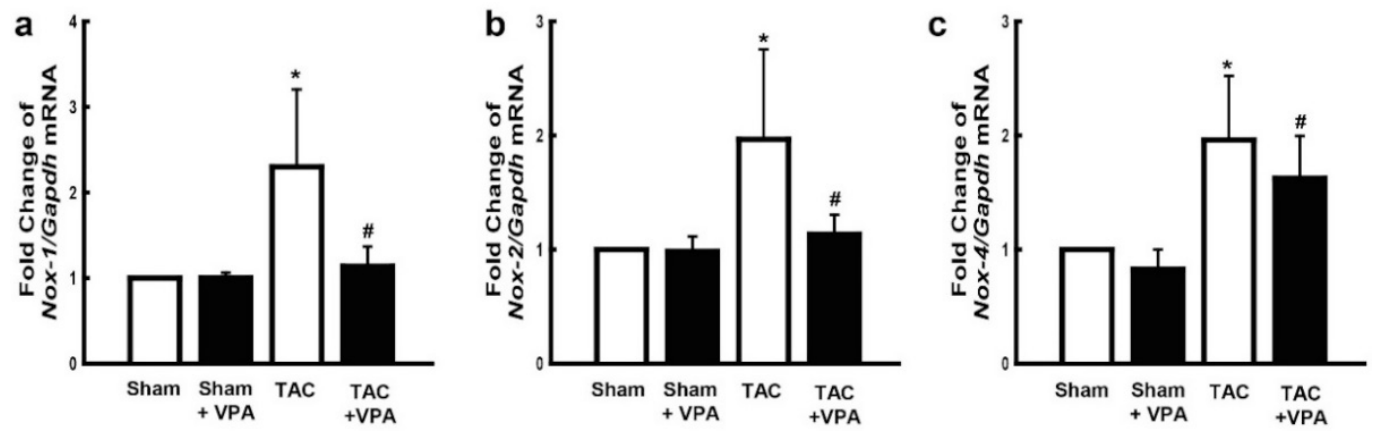

Fig. 4. Effect of VPA on oxidative stress. mRNA expression of NADPH oxidase subunits Nox-1 (a), Nox-2 (b), and Nox-4(c), oxidative stress markers, was determined by qRT-PCR. The TAC rats presented increased expression of Nox-1, -2 , and -4 mRNA, which was decreased with VPA treatment in TAC rats. Data are shown as mean \pm SE of six independent experiments $\left(* p<0.05\right.$ vs. sham, ${ }^{*} p<0.05$ vs. TAC).

a
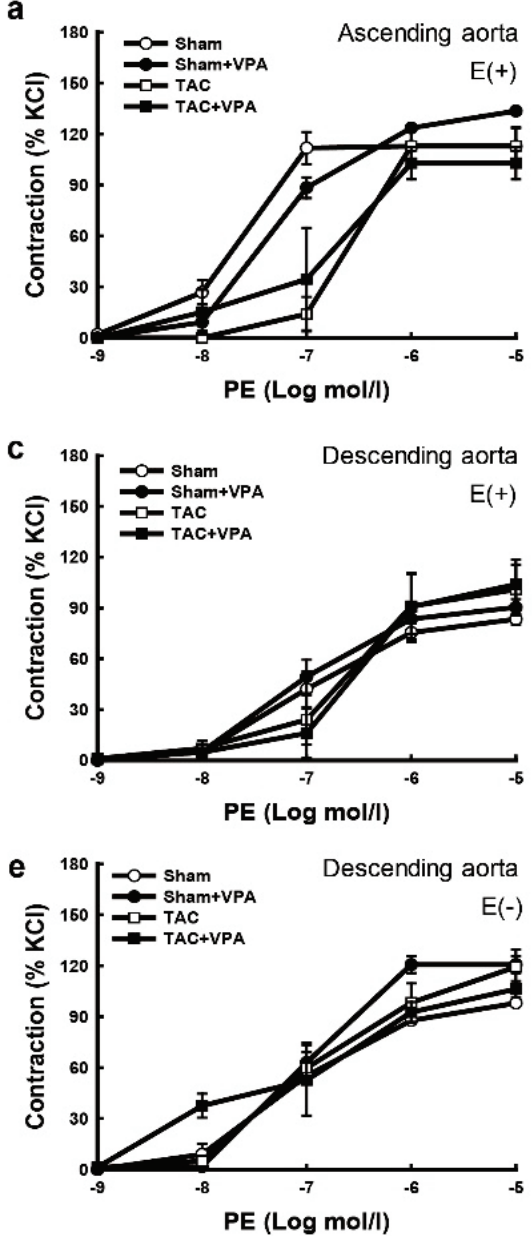

b

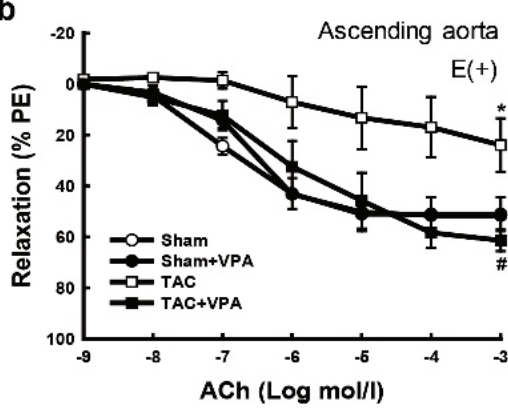

d
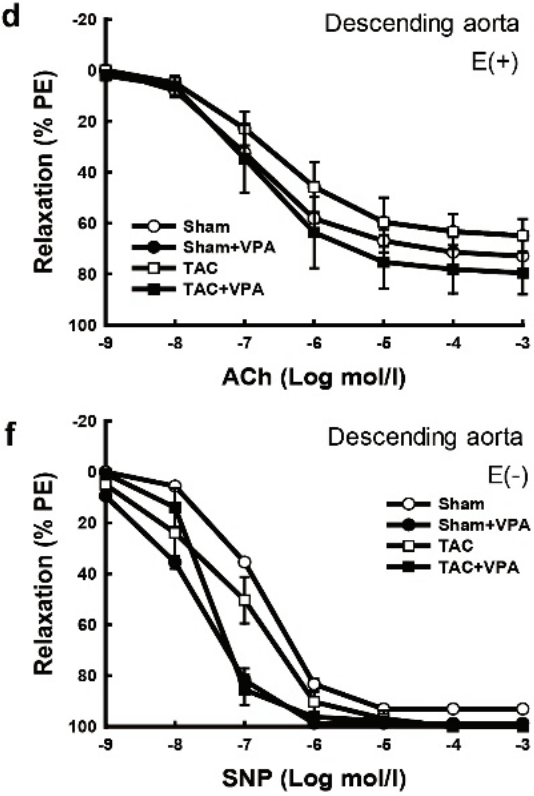

Fig. 5. Effects of VPA on aortic ring contraction and relaxation. Vascular contractile and relaxant responses were analyzed in the ascending (a and b) and descending thoracic (c to f) aortic rings. Phenylephrine (PE) was cumulatively added for vascular contraction in the aortic rings with intact (a and $\mathbf{d}$ ) or denuded (e) endothelium, whereas acetylcholine (ACh) and sodium nitroprusside (SNP) for vasorelaxation in the aortic rings with intact (b and $\mathbf{d}$ ) and denuded (f) endothelium, respectively. Developed tension is expressed as the percent of maximal contraction to $50 \mathrm{mmol} / \mathrm{l} \mathrm{KCl}$. Furthermore, developed relaxation is expressed as the percent of maximal contraction to PE. In the ascending aorta with intact endothelium, there was a significant decrease in the relaxation response, and VPA treatment considerably increased the relaxation response in TAC rats (b). Neither TAC nor VPA affected descending thoracic aortic ring contraction and relaxation. Data are shown as mean \pm SE of six independent experiments $(* \mathrm{p}<0.05$ vs. sham, ${ }^{\#} \mathrm{p}<0.05$ vs. TAC).

\section{Discussion}

In the present study, we demonstrate that HDAC inhibitor, VPA, has cardiac and protective effects in rats with pressure overload cardiac hypertrophy induced by TAC. Our results showed that VPA treatment attenuated the increased expression of hypertrophic, fibrotic, and oxidative stress genes and recovered the decreased relaxation response of ascending aorta with intact endothelium in pressure overload cardiac hypertrophic rats.

Studies have shown that HDAC inhibitors act via the direct regulation of cardiac protective effect, and hence, can be used to treat several heart diseases including cardiac hypertrophy and fibrosis (Cardinale et al. 2010, Kee et al. 2013, Lee et al. 2013, Kang et al. 
2015, Ooi et al. 2015, Lee et al. 2016). We identified that HDAC inhibitor, VPA, restores LV remodeling and improves heart function (Fig. 1). Cardiac hypertrophy is characterized by increase in heart mass, hypertrophy of cardiomyocyte, and up-regulation the expression of hypertrophic biomarker genes such as Nppa and Nppb. In our study, VPA inhibited the increase in heart weight (Fig. 1), attenuated histological hypertrophic changes, and down-regulated the expression of Nppa and Nppb (Fig. 2).

Cardiac fibrosis is accompanied with collagen deposition and fibrosis of myocardium. Furthermore, fibrosis might be the key factor of hypertrophied hearts under hypertensive condition (Diez 2007). Cardiac hypertrophy and fibrosis consequently increased left ventricular stiffness, resulting in cardiac remodeling in LV. Class I HDACs can regulate cardiac fibrosis. Class I HDAC inhibition prevents the growth of extracellular matrix-producing fibroblasts in the myocardium of congestive heart failure animal model (Nural-Guvener $e t$ al. 2014) and reduces fibrosis in the hearts of angiotensin II-infusion heart dysfunction rats (Williams et al. 2014). In contrast, HDAC6, a class IIb HDAC, controls expression of fibrosis-related genes such as $\alpha$-smooth muscle actin in heart dysfunction rats (Tao et al. 2016). Although HDAC inhibition appears to attenuate several pathogenic mechanisms that control heart failure, the precise mechanisms are still uncertain (McKinsey 2011). In the present study, VPA attenuates histological fibrotic changes and mRNA expression of Collagen-1, -3, and Fibronectin (Fig. 3). Meaning, HDAC inhibitor suppress left ventricle stiffness and regress cardiac hypertrophy.

During cardiac hypertrophy, cardiomyocytes are activated (Maulik and Kumar 2012) and hypertrophic response increases oxidative stress (Cardinale et al. 2010). We also demonstrated increased mRNA expression of $N A D P H$ oxidase subunits in the TAC rats, which was decreased with VPA treatment (Fig. 4). This confirms the role of HDAC inhibitor on oxidative stress during pressure overload cardiac hypertrophy in the rats.

The organ bath experiment to investigate aorta contraction and relaxation responses was conducted using the thoracic aorta cut into $4 \mathrm{~mm}$ rings. So far, the thoracic aorta is known as the descending thoracic aorta, which is completely different from the ascending aorta. Pressure overload cardiac hypertrophic rat model was obtained by TAC to represent patients with hypertensive heart disease, aortic stenosis, and hypertrophic cardiomyopathy. Starting with the pressure stress on the ascending aortic wall, LV hypertrophy is induced as compensatory response to hemodynamic pressure overload and eventually leads to heart failure. Therefore, to the best of our knowledge, we provide the evidence that organ bath experiment should be conducted with the ascending aorta and descending thoracic aorta separately in hypertensive animal models and of course analyzed individually, as the aorta wall and endothelium stress injury may be different. Surprisingly, there was a significant decrease in the relaxation of ascending aorta with intact endothelium, and VPA treatment considerably increased the relaxation of ascending aorta with intact endothelium in TAC rats (Fig. 5b). This was not observed in the descending thoracic aorta, irrespective of whether the endothelium was intact (Fig. 5d) or denuded (Fig. 5f).

In summary, the results of this study reveal that VPA restores LV remodeling; improves heart function; attenuates the increased expression of hypertrophic, fibrotic, and oxidative stress related genes; and recovers endothelium dysfunction in pressure overload cardiac hypertrophic rats. Therefore, the HDAC inhibitor, VPA, has cardiac and vascular protective effects in rats with pressure overload cardiac hypertrophy. Furthermore, organ bath experiment for aorta tension should be performed separately for ascending aorta and descending thoracic aorta.

\section{Conflict of Interest}

There is no conflict of interest.

\section{Acknowledgements}

This research was supported by the Kyungpook National University Research Fund, 2017 (Grant No. 201714940000). We appreciate Mr. Jun Woo Cho for his excellent technical support in echocardiography.

\section{References}

CARDINALE JP, SRIRAMULA S, PARIAUT R, GUGGILAM A, MARIAPPAN N, ELKS CM, FRANCIS J: HDAC inhibition attenuates inflammatory, hypertrophic, and hypertensive responses in spontaneously hypertensive rats. Hypertension 56: 437-444, 2010. 
CHEN S, SU J, WU K, HU W, GARDNER DG, CHEN D: Early captopril treatment prevents hypertrophy-dependent gene expression in hearts of SHR. Am J Physiol Regul Integr Comp Physiol 274: R1511-R1517, 1998.

CHO YK, EOM GH, KEE HJ, KIM H-S, CHOI W-Y, NAM K-I, MA JS, KOOK H: Sodium valproate, a histone deacetylase inhibitor, but not captopril, prevents right ventricular hypertrophy in rats. Circ J 74: 760-770, 2010.

DIEZ J: Mechanisms of cardiac fibrosis in hypertension. J Clin Hypertens (Greenwich) 9: 546-550, 2007.

GOMES AC, FALCAO-PIRES I, PIRES AL, BRAS-SILVA C, LEITE-MOREIRA AF: Rodent models of heart failure: an updated review. Heart Fail Rev 18: 219-249, 2013.

GURVICH N, TSYGANKOVA OM, MEINKOTH JL, KLEIN PS: Histone deacetylase is a target of valproic acidmediated cellular differentiation. Cancer Res 64: 1079-1086, 2004.

IYER A, FENNING A, LIM J, LE GT, REID RC, HALILI MA, FAIRLIE DP, BROWN L: Antifibrotic activity of an inhibitor of histone deacetylases in DOCA-salt hypertensive rats. Br J Pharmacol 159: 1408-1417, 2010.

KANG SH, SEOK YM, SONG MJ, LEE HA, KURZ T, KIM I: Histone deacetylase inhibition attenuates cardiac hypertrophy and fibrosis through acetylation of mineralocorticoid receptor in spontaneously hypertensive rats. Mol Pharmacol 87: 782-791, 2015.

KEE HJ, BAE EH, PARK S, LEE KE, SUH SH, KIM SW, JEONG MH: HDAC inhibition suppresses cardiac hypertrophy and fibrosis in DOCA-salt hypertensive rats via regulation of HDAC6/HDAC8 enzyme activity. Kidney Blood Press Res 37: 229-239, 2013.

KEE HJ, SOHN IS, NAM KI, PARK JE, QIAN YR, YIN Z, AHN Y, JEONG MH, BANG YJ, KIM N, KIM JK, KIM KK, EPSTEIN JA, KOOK H: Inhibition of histone deacetylation blocks cardiac hypertrophy induced by angiotensin II infusion and aortic banding. Circulation 113: 51-59, 2006.

LEE E, SONG MJ, LEE HA, KANG SH, KIM M, YANG EK, LEE DO Y, RO S, CHO JM, KIM I: Histone deacetylase inhibitor, CG200745, attenuates cardiac hypertrophy and fibrosis in DOCA-induced hypertensive rats. Korean J Physiol Pharmacol 20: 477-485, 2016.

LEE HA, LEE DY, CHO HM, KIM SY, IWASAKI Y, KIM IK: Histone deacetylase inhibition attenuates transcriptional activity of mineralocorticoid receptor through its acetylation and prevents development of hypertension. Circ Res 112: 1004-1012, 2013.

LUO T, CHEN B, WANG X: 4-PBA prevents pressure overload-induced myocardial hypertrophy and interstitial fibrosis by attenuating endoplasmic reticulum stress. Chem Biol Interact 242: 99-106, 2015.

MA J, LUO T, ZENG Z, FU H, ASANO Y, LIAO Y, MINAMINO T, KITAKAZE M: Histone deacetylase inhibitor phenylbutyrate exaggerates heart failure in pressure overloaded mice independently of HDAC inhibition. Sci Rep 6: 34036, 2016.

MAULIK SK, KUMAR S: Oxidative stress and cardiac hypertrophy: a review. Toxicol Mech Methods 22: 359-366, 2012.

MCKINSEY TA: Targeting inflammation in heart failure with histone deacetylase inhibitors. Mol Med 17: 434-441, 2011.

NURAL-GUVENER HF, ZAKHAROVA L, NIMLOS J, POPOVIC S, MASTROENI D, GABALLA MA: HDAC class I inhibitor, mocetinostat, reverses cardiac fibrosis in heart failure and diminishes CD90+ cardiac myofibroblast activation. Fibrogenesis Tissue Repair 7: 10, 2014.

OOI JY, TUANO NK, RAFEHI H, GAO XM, ZIEMANN M, DU XJ, EL-OSTA A: HDAC inhibition attenuates cardiac hypertrophy by acetylation and deacetylation of target genes. Epigenetics 10: 418-430, 2015.

ROCKMAN HA, ROSS RS, HARRIS AN, KNOWLTON KU, STEINHELPER ME, FIELD LJ, ROSS J, CHIEN KR: Segregation of atrial-specific and inducible expression of an atrial natriuretic factor transgene in an in vivo murine model of cardiac hypertrophy. Proc Natl Acad Sci U S A 88: 8277-8281, 1991.

SIMKO F, PECHANOVA O, REPOVA K, AZIRIOVA S, KRAJCIROVICOVA K, CELEC P, TOTHOVA L, VRANKOVA S, BALAZOVA L, ZORAD S, ADAMCOVA M: Lactacystin-induced model of hypertension in rats: effects of melatonin and captopril. Int J Mol Sci 18, 2017.

TAO H, YANG JJ, HU W, SHI KH, LI J: HDAC6 Promotes cardiac fibrosis progression through suppressing RASSF1A expression. Cardiology 133: 18-26, 2016. 
TAVAKOLI R, NEMSKA S, JAMSHIDI P, GASSMANN M, FROSSARD N: Technique of minimally invasive transverse aortic constriction in mice for induction of left ventricular hypertrophy. J Vis Exp 127: 2017.

WILLIAMS SM, GOLDEN-MASON L, FERGUSON BS, SCHUETZE KB, CAVASIN MA, DEMOS-DAVIES K, YEAGER ME, STENMARK KR, MCKINSEY TA: Class I HDACs regulate angiotensin II-dependent cardiac fibrosis via fibroblasts and circulating fibrocytes. J Mol Cell Cardiol 67: 112-125, 2014.

YOON S, EOM GH: HDAC and HDAC Inhibitor: from cancer to cardiovascular diseases. Chonnam Med J 52: 1-11, 2016.

YUAN Y, PENG W, LIU Y, XU Z: Palmatine attenuates isoproterenol-induced pathological hypertrophy via selectively inhibiting HDAC2 in rats. Int J Immunopathol Pharmacol 30: 406-412, 2017. 